\title{
A Psycholinguistic Analysis of Responses to Live-Stream Suicides on Social Media
}

\author{
Ang Li 1,2,3,*(D), Dongdong Jiao ${ }^{4}$, Xingyun Liu ${ }^{2}$, Jiumo Sun ${ }^{2}$ and Tingshao Zhu ${ }^{2, *}$ \\ 1 Department of Psychology, Beijing Forestry University, Beijing 100083, China \\ 2 Institute of Psychology, Chinese Academy of Sciences, Beijing 100101, China \\ 3 Black Dog Institute, University of New South Wales, Sydney 2031, Australia \\ 4 National Computer System Engineering Research Institute of China, Beijing 100083, China \\ * Correspondence: angli@bjfu.edu.cn (A.L.); tszhu@psych.ac.cn (T.Z.)
}

Received: 10 July 2019; Accepted: 8 August 2019; Published: 9 August 2019

check for updates

\begin{abstract}
Live-stream suicide has become an emerging public health problem in many countries. Regular users are often the first to witness and respond to such suicides, emphasizing their impact on the success of crisis intervention. In order to reduce the likelihood of suicide deaths, this paper aims to use psycholinguistic analysis methods to facilitate automatic detection of negative expressions in responses to live-stream suicides on social media. In this paper, a total of 7212 comments posted on suicide-related messages were collected and analyzed. First, a content analysis was performed to investigate the nature of each comment (negative or not). Second, the simplified Chinese version of the LIWC software was used to extract 75 psycholinguistic features from each comment. Third, based on 19 selected key features, four classification models were established to differentiate between comments with and without negative expressions. Results showed that $19.55 \%$ of 7212 comments were recognized as "making negative responses". Among the four classification models, the highest values of Precision, Recall, F-Measure, and Screening Efficacy reached 69.8\%, 85.9\%, 72.9\%, and $47.1 \%$, respectively. This paper confirms the need for campaigns to reduce negative responses to live-stream suicides and support the use of psycholinguistic analysis methods to improve suicide prevention efforts.
\end{abstract}

Keywords: live-stream suicide; psycholinguistic analysis; social media; Weibo

\section{Introduction}

Suicide is a major public health problem that can have harmful effects on individuals, families, and communities [1-6]. Suicidal people are often overwhelmed by feelings of hopelessness and social disconnection [7]. Therefore, the provision of crisis intervention, support, and assistance for suicidal people is essential to reduce their mortality risk [8], suggesting the importance of improving responses to suicides.

In recent years, live-stream suicide has become an emerging public health problem in many countries. On the internet, registered users are allowed to express their thoughts and feelings to a massive audience in real-time, and some of them even have used the internet platform to live stream their suicides (commonly known as live-stream suicide). Since there is not yet a consensus on the definition of live-stream suicide, in this study, it is defined as making a self-disclosure of suicidality (including suicidal ideation, suicide plans and suicide attempts) on the internet without time delays. Between 2003 and 2016, at least 193 relevant incidents have been reported in China [9]. Of these incidents, the majority took place on social media sites. When suicidal people disclose their suicidality on the internet (posting suicide-related messages), other regular users are likely to be the first to witness and respond to such suicides (posting comments on suicide-related messages). If 
they react appropriately, the likelihood of death may be reduced. However, a number of previous studies revealed the fact that users may react to live-stream suicides negatively. For example, Fu et al. examined a suicide incident that happened on Sina Weibo and analyzed 5971 generated posts [10]. Of these posts, $23.4 \%$ were identified as "cynical and indifferent comments". Li et al. collected and analyzed 4969 Weibo posts with suicide-related keywords. They found that $35.42 \%$ and $5.17 \%$ of the analyzed posts were associated with stigmatizing attitudes and negative behavioral intentions (e.g., refusing to offer help and encouraging suicide), respectively [11]. Ma et al. investigated six suicide incidents, and recognized "making cynical or indifferent comments, 'like' and incitement" as a major type of the response [12]. O'Dea et al. explored the first replies to suicide-related posts on Twitter and found that $23 \%$ of them reflected "dismissive or encouraging of the suicide" [13]. Results of these studies suggest that it is necessary to filter out such negative responses to reduce the likelihood of suicide death. However, users who witness live-stream suicides often post thousands of comments on suicide-related messages within several hours after disclosure of suicidality. The sheer volume of data makes it difficult for human coders to determine whether each comment reflect negative expressions or not. Therefore, there is a dire need for automatic detection of negative expressions in responses to live-stream suicides.

An examination of the words that people use in everyday language can provide insight into their psychological profiles [14]. A few recent studies found psycholinguistic characteristics of stigmatizing expressions in social media posts $[15,16]$, which suggests that the use of psycholinguistic analysis methods may improve our ability to automatically detect negative responses to mental health problems. However, these previous studies were limited by insufficient data collection and inadequate investigation of negative responses. Specifically, in these studies, researchers searched data using a set of keywords. As a result, such collected data may only reflect general opinions on a certain topic rather than actual responses. Moreover, researchers focused solely on stigmatizing attitudes, and dismissed other types of negative responses (e.g., negative behaviors/behavioral intentions). Therefore, additional analyses are necessary.

This study aims to examine actual responses to live-stream suicides on social media (Sina Weibo) and proposes the use of psycholinguistic analysis methods to establish computational models for automatic detection of negative expressions in responses.

\section{Materials and Methods}

The study was conducted in accordance with the Declaration of Helsinki. The protocol was approved by the Institutional Review Board at the Institute of Psychology, Chinese Academy of Sciences (protocol number: H09036 and H15009). The research process consisted of the following steps Figure 1: (i) Data collection, (ii) data pre-processing, and (iii) data analysis. Participant informed consent was not required for analyzing publicly available information $[17,18]$. To protect the participant privacy, personally identifiable information (including usernames and real names) was excluded from the analysis.

\subsection{Data Collection}

First, four incidents of live-stream suicides, which took place on Sina Weibo, were selected. Similar to Twitter, Sina Weibo is a free social media site that enables registered users to communicate and interact with others in real-time using posts. Although some users opt to privatize their accounts, the majority of the Weibo content is publicly available for viewing and downloading. To easily access and analyze data, in this study, we only focused on Sina Weibo rather than other internet platforms. According to a previous study [9], 30 relevant incidents that took place on Sina Weibo were identified. After that, the Weibo accounts associated with identified incidents were searched and found. To collect reliable and valid data, a further scrutiny of the Weibo accounts occurred to exclude the following: (i) Accounts that have been deleted, (ii) accounts with suicide-related posts deleted, and (iii) accounts with very few comments which were posted on suicide-related posts during the first $12 \mathrm{~h}$ 
after disclosure of suicidality (i.e., release of the first suicide-related post). As a result, a total of four target accounts were included for further analysis Table 1.

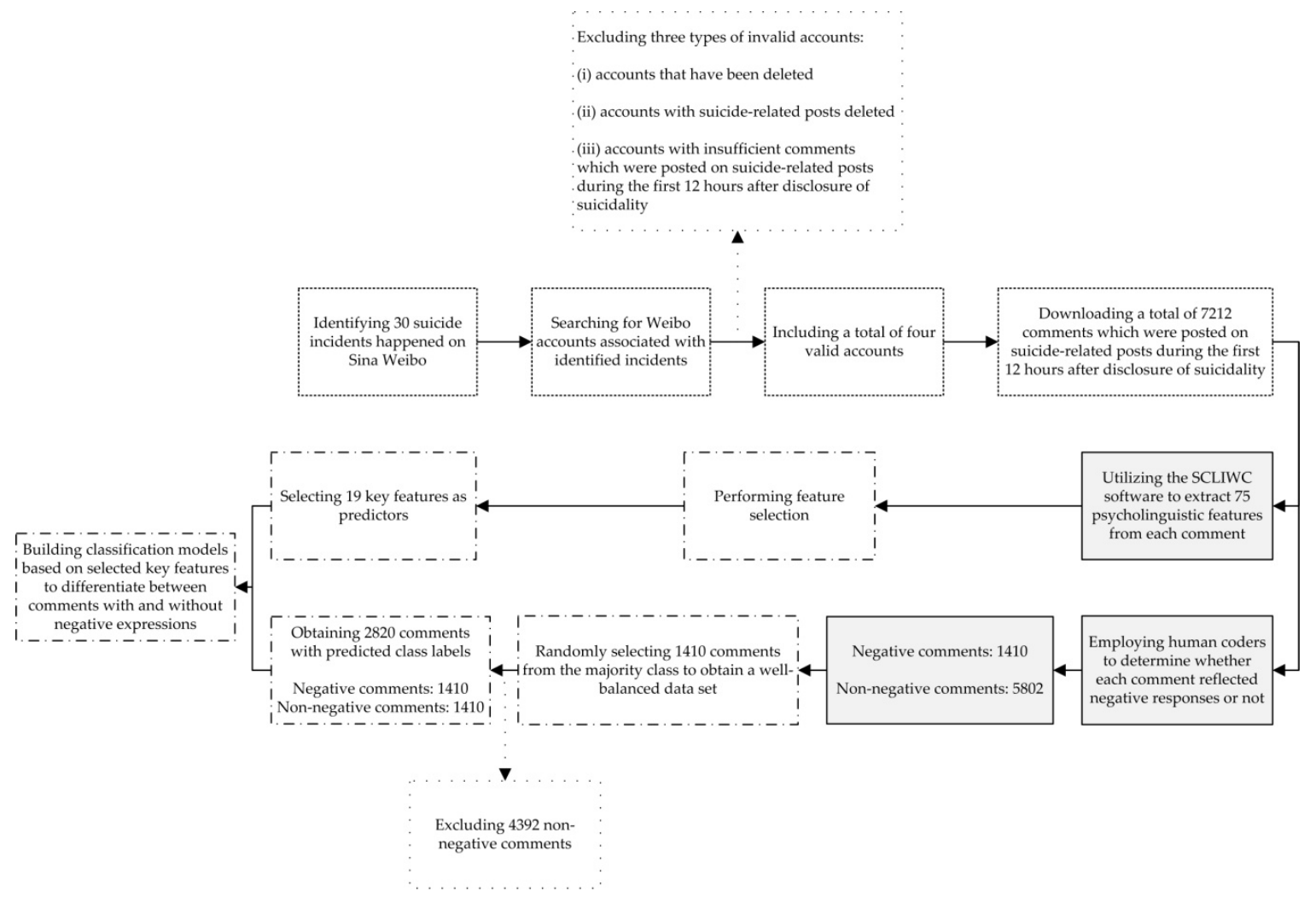

Figure 1. Research process.

Table 1. Details of suicide incidents.

\begin{tabular}{|c|c|c|c|c|}
\hline & Incident 1 & Incident 2 & Incident 3 & Incident 4 \\
\hline Time & 12/07/2012 21:05 & 16/02/2013 23:57 & 09/12/2013 17:36 & 19/02/2016 19:57 \\
\hline Location & Sichuan & Beijing & Shandong & Shanghai \\
\hline Gender & Female & Female & Female & Male \\
\hline Age & 31 & 31 & 33 & 40 \\
\hline Suicide method & $\begin{array}{l}\text { Taking poisons \& } \\
\text { charcoal burning }\end{array}$ & $\begin{array}{c}\text { Jumping from a } \\
\text { high place }\end{array}$ & Drowning & Hanging \\
\hline Suicide cause & Relationship breakup & Mental illness & Work-related stress & Mental illness \\
\hline Result & Rescued & Died & Died & Died \\
\hline
\end{tabular}

Second, for each target account, the corresponding comments were downloaded and analyzed. Since the duration of suicidal crises could be extended over several hours [19-21], in this study, responses were recognized as commenting on suicide-related posts during the first $12 \mathrm{~h}$ after release of the first suicide-related post. Finally, a total of 7212 comments were obtained.

\subsection{Data Pre-Processing}

First, to get predicted class labels for data modeling, two human coders were recruited to investigate the nature of each comment (negative responses or not). The coding framework was developed on the basis of expert consensus and available evidence. In specific, one researcher reviewed a number of relevant studies [10-13] and performed an inductive analysis of all 7212 comments to construct an initial framework. After that, two recruited coders gained a deep understanding of the initial framework and gave suggestions for its amendment. Finally, the initial framework was amended 
accordingly, and the formal framework was established in Table 2. By using the formal framework, two coders analyzed all 7212 comments independently. The levels of coding consistency between coders was evaluated by computing Cohen's $\kappa$ coefficients. If any inconsistency arises, a third researcher's opinion can resolve this issue.

Table 2. Coding framework.

\begin{tabular}{|c|c|c|}
\hline Category & Definition & Example Weibo Post \\
\hline Providing social support & $\begin{array}{l}\text { Expressing care and compassion, or } \\
\text { providing information, advice, } \\
\text { and resources }\end{array}$ & $\begin{array}{c}\text { "I hope you are ok! Don't die" } \\
\text { "You should get professional help now ..." } \\
\text { "My mom is an expert in this field! Don't die!! I can } \\
\text { ask her to help you!!!" }\end{array}$ \\
\hline Calling for help & Calling police and other users for help & $\begin{array}{l}\text { "Call the police!" } \\
\text { "Is there anyone who knows his address and can go } \\
\text { to his place to help him? ..." }\end{array}$ \\
\hline Expressing shock & Feeling surprised and upset & "What's up, don't scare me" \\
\hline Making negative responses & $\begin{array}{l}\text { Expressing cynical, dismissive, and } \\
\text { indifferent attitudes, or refusing to offer } \\
\text { help and encouraging suicide }\end{array}$ & $\begin{array}{l}\text { "Is there anything wrong with your brain? Why } \\
\text { not to kill yourself quietly ..." } \\
\text { "It is her last wishes, Why do you want to stop her" } \\
\text { "If you want to kill yourself, please be quick ..." }\end{array}$ \\
\hline Unspecified & $\begin{array}{l}\text { Replying with unspecified intent } \\
\text { or meaning }\end{array}$ & $\begin{array}{l}\text { “+G" } \\
\text { “........" } \\
\text { "Sigh" }\end{array}$ \\
\hline
\end{tabular}

Second, to get predictors for data modeling, the simplified Chinese version of the LIWC software (SCLIWC) (developed by the Computational Cyber-Psychology Lab at the Institute of Psychology, Chinese Academy of Sciences; website: http://ccpl.psych.ac.cn/textmind/) was utilized to extract psycholinguistic features from each comment. SCLIWC is a reliable and valid text analysis tool, which can be used to automatically estimate the words frequency in different psycholinguistic categories (e.g., emotional, cognitive, and other structural categories) [22]. Therefore, a total of 75 psycholinguistic features can be obtained for each comment.

\subsection{Data Analysis}

To filter out negative responses that may be harmful to suicidal users, in this study, all 7212 comments were classified into two groups, including the negative group (i.e., comments related to "making negative responses") and non-negative group (i.e., comments related to any one of other four categories). The WEKA software (Version 3.8.1) was utilized to build a series of classification models to differentiate between comments reflecting negative and non-negative responses.

First, to solve the class imbalance problem, a certain number of comments were randomly selected from the pool of the non-negative group. In this study, the data set is heavily imbalanced with $80 \%$ of data points being non-negative (negative group: 1410 comments; non-negative group: 5802 comments). In other words, in a binary classification problem, when $80 \%$ of data points belong to the false class (non-negative responses), a default prediction of false for all data points would lead to a classifier with high accuracy $(80 \%)$, even though the classifier has not learnt anything about the classification problem. Therefore, by using the method of simple random sampling, a total of 1410 comments (providing social support: 1190 comments; calling for help: 55 comments; expressing shock: 38 comments; unspecified: 127 comments) were randomly selected from the majority class (non-negative group) for further analysis. Between the sample (selected 1410 non-negative comments) and the population (all 5802 non-negative comments), there were no significant differences in the proportion of cases across the four non-negative subcategories. As a result, a well-balanced data set was obtained (negative group: 1410 comments; non-negative group: 1410 comments).

Second, to maximize the modeling performance, a series of key psycholinguistic features were selected as predictors. Since there is not yet a consensus on the selection of key psycholinguistic features, in this study, three different feature selection methods were used to explore key features 
automatically, including the gain ratio attribute evaluator (GRAE), the significance attribute evaluator (SAE), and the Chi-squared attribute evaluator (CAE). Based on such three methods, the weight of each feature was evaluated by measuring the gain ratio in terms of the class (GRAE), computing the probabilistic significance as a two-way function (feature-classes and classes-feature association) (SAE), and estimating the value of the Chi-squared statistic in terms of the class (CAE), respectively. As a result, according to different feature selection methods, all 75 features can be ranked with respect to their individual evaluations. In this research, key features were recognized as features ranked among the top 25 by all three methods (i.e., GRAE, SAE, and CAE). Key features were selected for use in the model construction.

Third, by using different algorithms (simple logistic regression, SLR; multilayer perceptron neural networks, MLPNN; support vector machine, SVM; random forest, RF), four classification models (SLR, MLPNN, SVM, and RF models) were established based on selected key features. 10-fold cross-validation was used to test each model. Specifically, the data set was randomly divided into ten subgroups with the same sample size. Each subgroup was used to test the model which was built on the other nine subgroups. After ten rounds of model training, the modeling results were integrated into a final model. Since coefficients in three of the four models (i.e., MLPNN, SVM, and RF models) could not indicate linear relationships between predictors and predicted class labels, regression coefficients in the SLR model were estimated to discover such relationships.

In this study, three indicators were used to measure the classification performance, including precision (number of true positives/number of instances predicted to be positive), recall (number of true positives/number of positive instances), and F-measure (a tradeoff between precision and recall).

Furthermore, an additional indicator (i.e., screening efficacy) was computed to evaluate the performance in reducing the workload of human coders for searching negative responses. In this study, the value of the screening efficacy can be estimated like this: (number of instances - number of instances predicted to be positive)/number of instances. For example, among 100 comments, 70 comments were predicted to be negative responses. Therefore, the value of the screening efficacy should be $30 \%$, implying that $30 \%$ of the workload of human coders can be reduced. In other words, only $70 \%$ of comments need to be analyzed by human coders.

\section{Results}

\subsection{Coding}

The outcomes of the coding task were shown in Table 3. Across different suicide incidents, the Cohen's $\kappa$ coefficients ranged from 0.83 to 0.89 , indicating a satisfying level of agreement [23].

Table 3. Coding results.

\begin{tabular}{cccccc}
\hline Category & Incident 1 & Incident 2 & Incident 3 & Incident 4 & Sum \\
\hline Providing social support & $671(41.06 \%)$ & $1583(79.75 \%)$ & $912(59.07 \%)$ & $1640(80.04 \%)$ & $4806(66.64 \%)$ \\
Calling for help & $87(5.32 \%)$ & $20(1.01 \%)$ & $111(7.19 \%)$ & $19(0.93 \%)$ & $237(3.29 \%)$ \\
Expressing shock & $41(2.51 \%)$ & $37(1.86 \%)$ & $28(1.81 \%)$ & $67(3.27 \%)$ & $173(2.40 \%)$ \\
Making negative responses & $697(42.66 \%)$ & $192(9.67 \%)$ & $371(24.03 \%)$ & $150(7.32 \%)$ & $1410(19.55 \%)$ \\
Unspecified & $138(8.45 \%)$ & $153(7.71 \%)$ & $122(7.90 \%)$ & $173(8.44 \%)$ & $586(8.13 \%)$ \\
\hline
\end{tabular}

Results showed that the proportions of comments reflecting positive responses (i.e., comments related to "providing social support" or "calling for help") ranged from $46.38 \%$ to $80.97 \%$, with an average of $69.93 \%$; while the proportions of comments reflecting negative responses ranged from $7.32 \%$ to $42.66 \%$, with an average of $19.55 \%$. 


\subsection{Differentiating between Negative/Non-Negative Responses}

A total of 19 key features were selected in Table 4 . The highest values of precision, recall, F-measure, and screening efficacy reached $69.8 \%, 85.9 \%, 72.9 \%$, and $47.1 \%$, respectively as shown in Table 5 . The logistic regression coefficients were shown in Table 6. Compared with non-negative comments, negative comments were related to an increased use of words in categories of Impersonal Pronouns $(\beta=1.05)$, Conjunctions $(\beta=3.15)$, Affective Processes $(\beta=0.83)$, Negative Emotion $(\beta=1.66)$, Exclusive $(\beta=2.28)$, and Death $(\beta=4.47)$; and were related to a decreased use of words in categories of Inclusive $(\beta=-0.99)$ and Assent $(\beta=-1.66)$.

Table 4. Psycholinguistic features selected by different methods.

\begin{tabular}{|c|c|c|c|}
\hline & Gain Ratio Attribute Evaluator & Significance Attribute Evaluator & Chi-Squared Attribute Evaluator \\
\hline 1 & Total Pronouns & Total Pronouns & Total Function Words \\
\hline 2 & Adverbs & Affective Processes & Affective Processes \\
\hline 3 & Cognitive Processes & Cognitive Processes & Adverbs \\
\hline 4 & Death & Adverbs & Total Pronouns \\
\hline 5 & Affective Processes & Exclusive & Cognitive Processes \\
\hline 6 & Total Function Words & Biological Processes & Biological Processes \\
\hline 7 & Exclusive & Death & Exclusive \\
\hline 8 & Biological Processes & Total Function Words & Death \\
\hline 9 & Body & Body & Auxiliary Verbs \\
\hline 10 & Impersonal Pronouns & Impersonal Pronouns & Impersonal Pronouns \\
\hline 11 & Common Verbs & Auxiliary Verbs & Conjunctions \\
\hline 12 & Conjunctions & Assent & Body \\
\hline 13 & Auxiliary Verbs & Conjunctions & Common Verbs \\
\hline 14 & Fillers & Common Verbs & Personal Pronouns \\
\hline 15 & Family & Personal Pronouns & Positive Emotion \\
\hline 16 & Swear Words & Fillers & Assent \\
\hline 17 & Third Pers Plural & Negative Emotion & Second Pers Singular \\
\hline 18 & Anger & Relativity & Negative Emotion \\
\hline 19 & Personal Pronouns & Family & Prepositions \\
\hline 20 & Negative Emotion & Second Pers Singular & Inclusive \\
\hline 21 & Assent & Inclusive & Discrepancy \\
\hline 22 & Nonfluencies & Humans & Health \\
\hline 23 & Inclusive & Positive Emotion & Relativity \\
\hline 24 & Social Processes & Social Processes & Humans \\
\hline 25 & Humans & Swear Words & Social Processes \\
\hline
\end{tabular}

Note. Features that dropped out of the top 25 were not listed.

Table 5. Performance of classification models in detecting negative responses.

\begin{tabular}{ccccc}
\hline & Precision & Recall & F-Measure & Screening Efficacy \\
\hline Simple Logistic Regression & $68.8 \%$ & $72.8 \%$ & $70.7 \%$ & $47.1 \%$ \\
Multilayer Perception Neural Networks & $66.2 \%$ & $79.6 \%$ & $72.3 \%$ & $39.9 \%$ \\
Support Vector Machine & $62.9 \%$ & $85.9 \%$ & $72.6 \%$ & $31.7 \%$ \\
Random Forest & $69.8 \%$ & $76.2 \%$ & $72.9 \%$ & $45.4 \%$ \\
\hline
\end{tabular}


Table 6. Regression coefficients in the Simple Logistic Regression model.

\begin{tabular}{ccc}
\hline & Predictors & $\boldsymbol{\beta}$ \\
\hline & Total Function Words & 2.31 \\
& Total Pronouns & 0.30 \\
& Impersonal Pronouns & 1.05 \\
& Common Verbs & -0.62 \\
& Auxiliary Verbs & 0.54 \\
Negative responses (1) & Adverbs & -0.24 \\
vs. & Conjunctions & 3.15 \\
Non-negative responses (0) & Humans & 2.21 \\
& Affective Processes & 0.83 \\
& Negative Emotion & 1.66 \\
& Inclusive & -0.99 \\
& Exclusive & 2.28 \\
& Biological Processes & -0.16 \\
& Body & 5.19 \\
& Death & 4.47 \\
& Assent & -1.66 \\
\hline
\end{tabular}

Note. Predictors without estimated $\beta$ values were not listed.

\section{Discussion}

In this paper, by using psycholinguistic analysis methods, we analyzed responses to live-stream suicides on Sina Weibo and discovered the ways in which negative responses are presented on social media.

First, there is a dire need for campaigns to reduce negative responses to live-stream suicide. Results of this study showed that, despite many positive comments, a large number of negative comments existed. According to the current study, the average proportion of negative comments reached $19.55 \%$, which is consistent with results of other previous studies $(\approx 23 \%)[10,13]$. Reducing suicide stigma and enhancing public awareness of suicide literacy may be the key to improving reactions to live-stream suicides fundamentally [11,16]. Social media campaigns might be helpful to address this concern. Specifically, social media enables registered users to bring personal experiences into the public domain, which might influence public attitudes, mainstream media, and even policy [24], such as the "Stigma Watch" program (https://www.sane.org/stigmawatch). Moreover, social media users are allowed to create their own social networks, which can be leveraged to facilitate the acceptance of received knowledge and then accelerate the change of individual attitude and behavior [25-27].

Second, the use of psycholinguistic analysis methods facilitates automatic detection of negative expressions in responses to live-stream suicides on social media. In this study, by using psycholinguistic analysis methods, computational models were established to differentiate between comments with and without negative expressions automatically. Results showed that the highest F-measure value reached $72.9 \%$. Compared with results of similar studies (F-measure: 37-83\%) [15,16,18,28-30], the classification performance is satisfying in this current study. Moreover, with the help of established classification models, the workload of human coders can also be reduced considerably (screening efficacy: $31.7 \%-47.1 \%$ ).

Third, the use of psycholinguistic analysis methods provides insight into how negative responses are presented on social media. According to the coding framework, in this study, negative responses included expressing negative attitudes (expressing cynical, dismissive, and indifferent attitudes) and manifesting negative behaviors/behavioral intentions (refusing to offer help and encouraging suicide). Results showed that negative responses were associated with an increased use of words in categories of Impersonal Pronouns (e.g., it, those), Conjunctions (e.g., but, whereas), and Exclusive (e.g., without, exclude), and were associated with a decreased use of words in the category of Inclusive (e.g., with, include), indicating a desire to make a distinction between people in one group and people from the other group $[14,31,32]$. Furthermore, negative responses were also related to an increased use of words 
in categories of Affective Processes (e.g., cried, abandon) and Negative Emotion (e.g., ugly, nasty), indicating a high level of negative emotion. Such psycholinguistic patterns might fit into two major elements of stigmatizing attitudes, including cognitive separating and emotional reactions [33], which is consistent with results of previous studies $[15,16]$. In addition, a decreased use of Assent-related words (e.g., agree, yes) indicated negative responses contained fewer positive assertions than non-negative responses. Apart from expressing negative attitudes, manifesting negative behaviors/behavioral intentions is another major type of the negative response. It might be the reason why negative responses were related to an increased use of words in the category of Death (e.g., kill, bury).

Limitations exist. First, only four suicide incidents were involved in this study. Although a large number of comments, the small number of incidents may limit the generalizability of the current study findings. Second, given that a number of live-stream suicides happened outside of Sina Weibo, different outcomes may be found on other internet platforms. Third, there is no evidence to confirm that responses to live-stream suicides are the same as responses to offline suicides. Fourth, social media users are not representative of the general population, and thus the outcomes may not be applicable to the general public. Finally, it is unclear if the findings in this research can also be applied to other types of language. In spite of these disadvantages, in this study, a non-intrusive method was used to analyze responses to live-stream suicides. As a result, the current research should have high ecological validity and is possible to indicate the actual reactions to live-stream suicides. In addition, this study did not just focus on what a comment says precisely, but how it is expressed. Therefore, the use of psycholinguistic analysis methodologies can be beneficial to enhance the validity of automatic detection of negative responses.

\section{Conclusions}

This paper confirms the need for campaigns to reduce negative responses to live-stream suicides and supports the use of psycholinguistic analysis methods to improve suicide prevention efforts.

Author Contributions: Conceptualization, A.L. and T.Z.; Methodology, A.L. and D.J.; Software, A.L. and D.J.; Formal analysis, A.L., D.J., X.L. and J.S.; Data curation, A.L. and T.Z.; Writing-Original draft preparation, A.L.; Writing-Review and editing, A.L. and T.Z.

Funding: This study was partially supported by the Key Research Program of the Chinese Academy of Sciences (No. ZDRW-XH-2019-4).

Acknowledgments: Not applicable.

Conflicts of Interest: The authors declare no conflict of interest.

\section{References}

1. World Health Organization. Preventing Suicide: A Global Imperative; World Health Organization: Geneva, Switzerland, 2014.

2. Cerel, J.; Jordan, J.R.; Duberstein, P.R. The impact of suicide on the family. Crisis 2008, 29, 38-44. [CrossRef] [PubMed]

3. Linke, S.; Wojciak, J.; Day, S. The impact of suicide on community mental health teams: Findings and recommendations. Psychiatr. Bull. 2002, 26, 50-52. [CrossRef]

4. Levine, H. Suicide and its impact on campus. New Dir. Stud. Serv. 2008, 121, 63-76. [CrossRef]

5. Carr, R.B. When a soldier commits suicide in Iraq: Impact on unit and caregivers. Psychiatry 2011, 74, 95-106. [CrossRef] [PubMed]

6. Seeman, M.V. The impact of suicide on co-patients. Psychiatr. Q. 2015, 86, 449-457. [CrossRef] [PubMed]

7. Joiner, T.E., Jr.; Brown, J.S.; Wingate, L.R. The psychology and neurobiology of suicidal behavior. Annu. Rev. Psychol. 2005, 56, 287-314. [CrossRef] [PubMed]

8. Ross, A.M.; Kelly, C.M.; Jorm, A.F. Re-development of mental health first aid guidelines for suicidal ideation and behaviour: A delphi study. BMC Psychiatry 2014, 14, 241. [CrossRef] [PubMed]

9. Li, A.; Huang, X.; Zhu, T. A systematic analysis of online broadcasts of suicidality in China. Asia Pac. Psychiatry 2018, 10, e12302. [CrossRef] 
10. Fu, K.W.; Cheng, Q.; Wong, P.W.; Yip, P.S. Responses to a self-presented suicide attempt in social media: A social network analysis. Crisis 2013, 34, 406-412. [CrossRef]

11. Li, A.; Huang, X.; Hao, B.; O’Dea, B.; Christensen, H.; Zhu, T. Attitudes towards suicide attempts broadcast on social media: An exploratory study of Chinese microblogs. Peer] 2015, 3, e1209. [CrossRef]

12. Ma, J.; Zhang, W.; Harris, K.; Chen, Q.; Xu, X. Dying online: Live broadcasts of Chinese emerging adult suicides and crisis response behaviors. BMC Public Health 2016, 16, 774. [CrossRef] [PubMed]

13. O’Dea, B.; Achilles, M.R.; Larsen, M.E.; Batterham, P.J.; Calear, A.L.; Christensen, H. The rate of reply and nature of responses to suicide-related posts on Twitter. Internet Interv. 2018, 13, 105-107. [CrossRef] [PubMed]

14. Pennebaker, J.W.; Mehl, M.R.; Niederhoffer, K.G. Psychological aspects of natural language use: Our words, our selves. Annu. Rev. Psychol. 2003, 54, 547-577. [CrossRef] [PubMed]

15. Li, A.; Jiao, D.; Zhu, T. Detecting depression stigma on social media: A linguistic analysis. J. Affect. Disord. 2018, 232, 358-362. [CrossRef] [PubMed]

16. Li, A.; Huang, X.; Jiao, D.; O’Dea, B.; Zhu, T.; Christensen, H. An analysis of stigma and suicide literacy in responses to suicides broadcast on social media. Asia Pac. Psychiatry 2018, 10, e12314. [CrossRef] [PubMed]

17. Reavley, N.J.; Pilkington, P.D. Use of Twitter to monitor attitudes toward depression and schizophrenia: An exploratory study. PeerJ 2014, 2, e647. [CrossRef] [PubMed]

18. O'Dea, B.; Wan, S.; Batterham, P.J.; Calear, A.L.; Paris, C.; Christensen, H. Detecting suicidality on Twitter. Internet Interv. 2015, 2, 183-188. [CrossRef]

19. Simon, O.R.; Swann, A.C.; Powell, K.E.; Potter, L.B.; Kresnow, M.J.; O'Carroll, P.W. Characteristics of impulsive suicide attempts and attempters. Suicide Life Threat. Behav. 2001, 32, 49-59. [CrossRef]

20. Hawton, K. Restricting access to methods of suicide: Rationale and evaluation of this approach to suicide prevention. Crisis J. Crisis Interv. Suicide Prev. 2007, 28, 4-9. [CrossRef]

21. Kattimani, S.; Sarkar, S.; Menon, V.; Muthuramalingam, A.; Nancy, P. Duration of suicide process among suicide attempters and characteristics of those providing window of opportunity for intervention. J. Neurosci. Rural Pract. 2016, 7, 566-570.

22. Zhao, N.; Jiao, D.; Bai, S.; Zhu, T. Evaluating the validity of simplified Chinese version of LIWC in detecting psychological expressions in short texts on social network services. PLoS ONE 2016, 11, e0157947. [CrossRef] [PubMed]

23. Landis, J.R.; Koch, G.G. The measurement of observer agreement for categorical data. Biometrics 1977, 33, 159-174. [CrossRef] [PubMed]

24. Betton, V.; Borschmann, R.; Docherty, M.; Coleman, S.; Brown, M.; Henderson, C. The role of social media in reducing stigma and discrimination. Br. J. Psychiatry 2015, 206, 443-444. [CrossRef] [PubMed]

25. Christakis, N.A.; Fowler, J.H. The spread of obesity in a large social network over 32 years. N. Engl. J. Med. 2007, 357, 370-379. [CrossRef] [PubMed]

26. Valente, T.W. Network interventions. Science 2012, 337, 49-53. [CrossRef] [PubMed]

27. Bond, R.M.; Fariss, C.J.; Jones, J.J.; Kramer, A.D.I.; Marlow, C.; Settle, J.E.; Fowler, J.H. A 61-million-person experiment in social influence and political mobilization. Nature 2012, 489, 295-298. [CrossRef] [PubMed]

28. Guan, L.; Hao, B.; Cheng, Q.; Yip, P.S.; Zhu, T. Identifying Chinese microblog users with high suicide probability using internet-based profile and linguistic features: Classification model. JMIR Ment. Health 2015, 2, e17. [CrossRef] [PubMed]

29. McManus, K.; Mallory, E.K.; Goldfeder, R.L.; Haynes, W.A.; Tatum, J.D. Mining Twitter data to improve detection of schizophrenia. AMIA Jt. Summits Transl. Sci. Proc. 2015, 2015, 122-126.

30. Jiang, K.; Gupta, R.; Gupta, M.; Calix, R.A.; Bernard, G.R. Identifying personal health experience Tweets with deep neural networks. In Proceedings of the 2017 39th Annual International Conference of the IEEE Engineering in Medicine and Biology Society (EMBC), Seogwipo, Korea, 11-15 July 2017.

31. Pennebaker, J.W.; King, L.A. Linguistic styles: Language us as an individual difference. J. Personal. Soc. Psychol. 1999, 77, 1296-1312. [CrossRef] 
32. Qiu, L.; Lu, J.; Ramsay, J.; Yang, S.; Qu, W.; Zhu, T. Personality expression in Chinese language use. Int. J. Psychol. 2017, 52, 463-472. [CrossRef]

33. Link, B.G.; Yang, L.H.; Phelan, J.C.; Collins, P.Y. Measuring mental illness stigma. Schizophr. Bull. 2014, 30, 511-541. [CrossRef] [PubMed]

(C) 2019 by the authors. Licensee MDPI, Basel, Switzerland. This article is an open access article distributed under the terms and conditions of the Creative Commons Attribution (CC BY) license (http://creativecommons.org/licenses/by/4.0/). 